[0212-7199 (2006) 23: 8; pp 385-386] ANALES DE MEDICINA INTERNA Copyright (C) 2006 ARAN EDICIONES, S.L.

AN. MED. InTERNA (Madrid) Vol. 23, N. ${ }^{\circ}$, pp. 385-386, 2006

\title{
Hemorragia digestiva por leiomiosarcoma de intestino delgado
}

\section{R. BAÑOS MADRID, A. ALBALADEJO MEROÑO, A. SERRANO JIMÉNEZ, M. ALAJARÍN CERVERA, A. VARGAS ACOSTA, F. ALBERCA DE LAS PARRAS, J. MOLINA MARTÍNEZ, F. CARBALLO}

Unidad de Endoscopia Digestiva. Servicio de Aparato Digestivo. Hospital Universitario Virgen de la Arrixaca. Murcia

GASTROINTESTINAL BLEEDING CAUSED BY SMALL BOWEL LEIOMYOSARCOMA

\begin{abstract}
RESUMEN
El leiomiosarcoma de intestino delgado es un tumor infrecuente en la práctica clínica. Presentamos el caso de un hombre de 50 años que ingresó en el hospital en dos ocasiones por hemorragia digestiva. En el segundo ingreso una enteroscopia permitió localizar en yeyuno un tumor. El diagnóstico anatomopatológico fue de un leiomiosarcoma de yeyuno de bajo grado. El paciente precisó cirugía con resección de la porción de yeyuno afectada.
\end{abstract}

PALABRAS CLAVE: Hemorragia digestiva. Leiomiosarcoma intestino delgado.

\section{ABSTRACT}

The small bowel leiomiosarcoma is a infrequent tumour in the clinical practice.We report the case of a 50 year old male admitted to the hospital on two occasions for gastrointestinal bleeding. On the second ocassion a push enteroscopy located in jejunum a tumour. The pathologic diagnosis was a low grade jejunal leiomyosarcoma. The patient required a surgical intervention with resection of the affected portion of the jejunum.

KEY WORDS: Gastrointestinal bleeding. Small bowel leiomyosarcoma.

Baños Madrid R, Albaladejo Meroño A, Serrano Jiménez, A, Alajarín Cervera M, Vargas Acosta A, Alberca de las Parras F, Molina Martínez. J, Carballo F. Hemorragia digestiva por leiomiosarcoma de intestino delgado. An Med Interna (Madrid) 2006; 23: 385-386.

\section{INTRODUCCIÓN}

Los tumores malignos de intestino delgado son raros, los adenocarcinomas, linfomas, tumores carcinoides y sarcomas representan la mayoría de las neoplasias malignas del intestino delgado y en conjunto solo constituyen el $1 \%$ de todas las neoplasias malignas gastrointestinales. El sarcoma más frecuente es el leiomiosarcoma que se encuentra habitualmente en yeyuno. Este tipo de tumor se puede manifestar como anemia crónica, dolor abdominal, síndrome constitucional, tumor palpable, obstrucción intestinal o de forma característica como hemorragia digestiva alta o baja dependiendo de su localización en intestino delgado (1).

\section{CASO APORTADO}

Varón de 50 años con antecedentes de hipertensión arterial en tratamiento y apendicectomía a los 25 años, consulta por epigastralgia, melenas y astenia desde hacía quince días. No refería disfagia, pirosis ni alteración del hábito intestinal.
La exploración física fue normal, no se palpaban masas ni visceromegalias en abdomen, ni existían signos de desnutrición.

En la analítica destacaba la presencia de anemia microcítica hipocroma con $\mathrm{Hgb}$ de $10 \mathrm{gr} / \mathrm{dl}$ y Hto del 33\%. Las radiografías de tórax y abdomen no mostraron alteraciones de interés. En la endoscopia digestiva alta se apreció un esófago normal, una cavidad gástrica sin restos hemáticos con una mucosa normal y erosiones sobre una mucosa edematosa en bulbo duodenal, se realizó toma de biopsias en antro y cuerpo informándose las biopsias como gastritis crónica sin actividad no detectándose helicobacter pylori. En la ecografía abdominal el hígado, la vesícula biliar y el páncreas eran de características normales.

Con el diagnóstico de hemorragia digestiva alta por duodenitis erosiva y anemia secundaria a perdidas digestivas es dado de alta para control ambulatorio con tratamiento con antisecretores. El paciente reingresa a las tres semanas por nuevo episodio de melenas, se realiza una nueva endoscopia digestiva alta apreciando un esófago, mucosa de cavidad gástrica y bulbo duodenal normal, con restos hemáticos aislados a nivel de la segunda porción de duodeno, por lo que se decide la realización de enteroscopia de pulsión apreciando en yeyuno proximal una tumoración, friable y ulcerada que estenosa parcialmente la luz de yeyuno y no permite el paso del endoscopio, 
pero si la toma de múltiples biopsias en los bordes del tumor. Se procedió a la realización de un tránsito intestinal en el que se evidenció en yeyuno proximal una estenosis corta, que permitía el paso del contraste (Fig. 1). Se completa el estudio de extensión realizando un TAC abdominal en el que se puso de manifiesto la presencia de una masa en yeyuno proximal sin objetivarse adenopatías locoregionales ni retroperitoneales de tamaño significativo, no se observaron lesiones focales hepáticas sugerentes de metástasis, ni otras alteraciones radiológicas valorables. Con el diagnóstico de leiomiosarcoma de bajo grado de intestino delgado y tras descartar extensión tumoral a distancia o invasión local en los estudios de imagen, se decide tratamiento quirúrgico con resección del tumor y posterior anastomosis duodeno-yeyunal. La evolución de la paciente fue satisfactoria no precisando tratamiento coadyuvante.

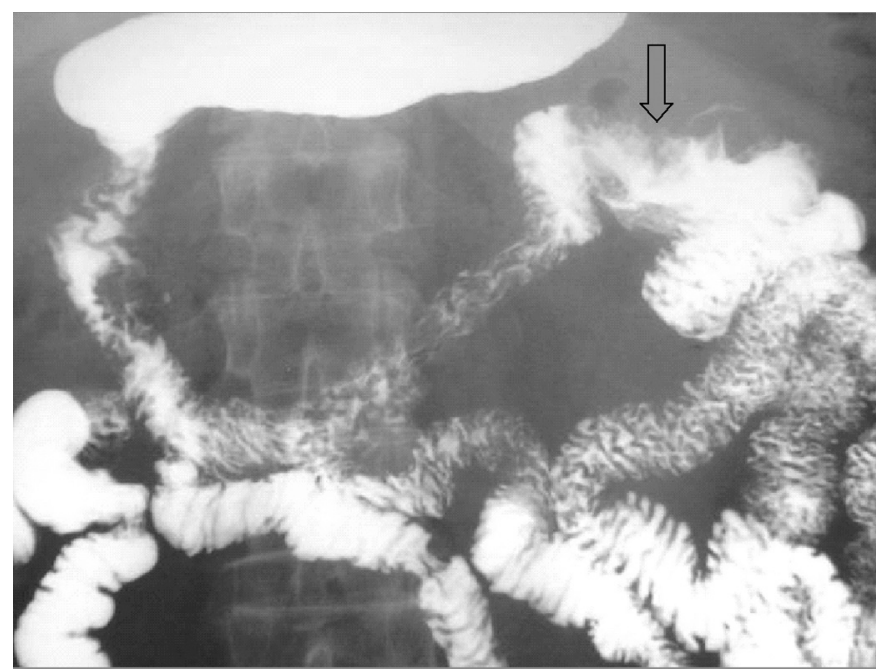

Fig. 1. Tránsito intestinal, a nivel de yeyuno proximal se aprecia una estenosis corta con borramiento de pliegues que permite el paso de contraste distalmente.

\section{DISCUSIÓN}

Los tumores malignos del intestino delgado más frecuentes son el adenocarcinoma (el más frecuente), tumor carcinoide, linfoma y el leiomiosarcoma. El leiomiosarcoma es un tumor submucoso extremadamente raro en intestino delgado, con una incidencia anual de 1 por 1.000 .000 , y suele presentarse en mujeres mayores de 50 años, se origina en el músculo liso intestinal y es más común en yeyuno. La presentación clí- nica es idéntica a la de otros tumores de intestino delgado (anemia, dolor abdominal, cuadro constitucional, obstrucción intestinal), destacando una mayor tendencia a experimentar hemorragia intestinal por necrosis del tumor y ulceración de la mucosa suprayacente $(1,2)$. En ausencia de diseminación a distancia o invasión local, los leiomiosarcomas pueden ser difíciles de distinguir de los leiomiomas, siendo imprescindible en esta situación el estudio anatomopatológico apreciando un mayor índice mitótico en los leiomiosarcomas. Los leiomiosarcomas habitualmente son tumores grandes, de más de 5 $\mathrm{cm}$, presentando un crecimiento hacia la superficie serosa, invadiendo el mesenterio y estructuras vecinas. Debido a que el intestino delgado solo es accesible a la endoscopia convencional hasta los primeros tramos de duodeno, el diagnóstico de la patología distal a la segunda porción de duodeno se retrasa de forma habitual hasta una fase muy avanzada de la enfermedad, esto es especialmente grave en la enfermedad tumoral que afecta al intestino delgado. Hasta no hace mucho la radiología baritada tradicional o la enteroclisis eran las pruebas complementarias de imagen más útiles. El diagnóstico definitivo era posible posteriormente con la enteroscopia o la cirugía, al permitir confirmar el diagnóstico con el estudio anatomopatológico, por último la tomografía computerizada nos permitía completar el estudio de extensión a tejidos adyacentes o valorar metástasis a distancia $(2,3)$. Actualmente con la aparición de la cápsula endoscópica y la enteroscopia de doble balón se ha revolucionado el diagnóstico y el tratamiento de las enfermedades del intestino delgado (4). Como en otras neoplasias malignas gastrointestinales el leiomiosarcoma de intestino delgado tiene como principal opción para lograr supervivencia a largo plazo el tratamiento quirúrgico, sobre todo cuando se hace en tumores diagnosticados en fase inicial. La curación se relaciona con la capacidad de resecar completamente el tumor siendo actualmente la supervivencia a 5 años para el leiomiosarcoma resecable superior al $50 \%$. Existen datos contradictorios con respecto al uso de quimioterapia o radioterapia neoadyuvante o paliativa. En aquellos pacientes con leiomiosarcoma de intestino irresecable el tratamiento de elección es la derivación del tránsito intestinal quirúrgicamente y la radioterapia, si además hay metástasis está indicada la cirugía paliativa y la quimio-radioterapia paliativa. Es por tanto actualmente el tratamiento quirúrgico el único con beneficios probados en el manejo de la enfermedad tumoral del intestino delgado. Los factores pronósticos están en relación con los márgenes de resección y la invasión del tumor local y a distancia (5-7).

\section{Bibliografía}

1. Gill SS, Heuman DM, Mihas AA. Small intestinal neoplasms. J Clin Gastroenterol 2001; 33: 267-282.

2. Buzday AD, Nazli O, Tansug T, Isguder AS. Primary tumors of the small bowel: diagnosis, treatment and prognostic factors. Hepatogastroenterology 2003; 50: 256-258.

3. Samaiya A, Deo SS, Thulkin S, Hazarika S, Kumar S, Parida DK, Shukla NK. An unusual presentation of a malignant jejunal tumor and a different management strategy. World J Surg Oncol 2005; 3: 3.

4. Zepeda-Gómez S, Nuncio JF, Maldonado H, Arista-Nasr J, Sánchez E, Schmulson M, et al. Gastrointestinal stromal tumors: clinical and patho-

logical analysis of 24 cases. Rev Invest Clin 2004; 56: 443-8.

5. Zhan J, Xin ZS, Zhong YQ, Zhang SN, Wang LY, Shu H, et al. Clinical analysis of primary small intestinal disease. A report of 309 cases. World J Gastroenterol 2004; 10: 2585-7.

6. Korman MU. Radiologic evaluation and staging of small intestine neoplasms. Eur J Radiol 2002; 42: 193-205.

7. Martínez-Ares D, González-Conde B, Yánez J, Estévez E, Arnal F, Lorenzo J, et al. Jejunal Leiomiosarcoma, a rare cause of obscure gastrointestinal bleeding diagnosis by gíreles capsule endoscopy. Surg Endosc 2004; 18: 554-6. 